\title{
Survival rate in nasopharyngeal carcinoma improved by high caseload volume: a nationwide population-based study in Taiwan
}

\author{
Ching-Chih Lee ${ }^{1,6,7}$, Tze-Ta Huang ${ }^{2,6}$, Moon-Sing Lee ${ }^{3,6}$, Yu-Chieh Su ${ }^{4,6}$, Pesus Chou ${ }^{7}$, Shih-Hsuan Hsiao ${ }^{1,6}$, \\ Wen-Yen Chiou ${ }^{3,6}$, Hon-Yi Lin ${ }^{3,6}$, Sou-Hsin Chien ${ }^{5,6^{*}}$ and Shih-Kai Hung ${ }^{3,6^{*}}$
}

\begin{abstract}
Background: Positive correlation between caseload and outcome has previously been validated for several procedures and cancer treatments. However, there is no information linking caseload and outcome of nasopharyngeal carcinoma (NPC) treatment. We used nationwide population-based data to examine the association between physician case volume and survival rates of patients with NPC.

Methods: Between 1998 and 2000, a total of 1225 patients were identified from the Taiwan National Health Insurance Research Database. Survival analysis, the Cox proportional hazards model, and propensity score were used to assess the relationship between 10-year survival rates and physician caseloads.
\end{abstract}

Results: As the caseload of individual physicians increased, unadjusted 10-year survival rates increased $(p<0.001)$. Using a Cox proportional hazard model, patients with NPC treated by high-volume physicians (caseload $\geq 35$ ) had better survival rates $(p=0.001)$ after adjusting for comorbidities, hospital, and treatment modality. When analyzed by propensity score, the adjusted 10-year survival rate differed significantly between patients treated by highvolume physicians and patients treated by low/medium-volume physicians ( $75 \%$ vs. 61\%; $p<0.001)$.

Conclusions: Our data confirm a positive volume-outcome relationship for NPC. After adjusting for differences in the case mix, our analysis found treatment of NPC by high-volume physicians improved 10-year survival rate.

\section{Introduction}

The fact that increased caseload is associated with better patient outcomes has been noted for three decades in many areas of health care, including acute myocardial infarction, many types of high-risk surgeries, and cancer treatment $[1,2]$. The "practice makes perfect" hypothesis may be valid for certain procedures such as open-heart and vascular surgery and "selective referral" may in part account for this phenomenon $[3,4]$. However, such a positive volume-outcome relationship is not well validated for other procedures. Only a few studies have examined the effect of physician caseload on treatment outcome for head and neck cancers $[5,6]$.

\footnotetext{
* Correspondence: shchien@tzuchi.com.tw; oncology158@yahoo.com.tw ${ }^{3}$ Department of Radiation Oncology, Buddhist Dalin Tzu Chi General Hospital, Chiayi, Taiwan

${ }^{5}$ Division of Plastic Surgery, Department of Surgery, Buddhist Dalin Tzu Chi General Hospital, Chiayi, Taiwan

Full list of author information is available at the end of the article
}

Taiwan has a high incidence of nasopharyngeal carcinoma (NPC): the annual incidence rate is 6.17 per 100,000 as compared with $<1$ per 100,000 in Western countries [7]. Radiotherapy or concurrent chemoradiotherapy (CCRT) is the principal treatment because NPC is anatomically inaccessible and highly sensitive to radiotherapy and chemotherapy [8].

Previous volume-outcome studies have shown improved treatment outcome in breast cancer, oral cancer, esophageal cancer, radical prostatectomy, and nephrectomy [5,9-11]. However, there is scant information on the volume-outcome relationship for NPC. The purpose of this study was to examine the relationship between physician caseload and survival rate in NPC using population-based data.

In most previous studies on the association between caseload and outcome, a Cox proportional hazards model or logistic regression was routinely used, raising

\section{Biomed Central}


the possibility that selection bias might still exist. Therefore, we evaluated the association between physician caseload and survival rate using population-based data, Cox regression analysis, and propensity score to minimize the effect of selection bias.

\section{Patients and methods}

The database contained a registry of contracted medical facilities, a registry of board-certified physicians, and monthly claims summary for all inpatient claims. Because these were de-identified secondary data, this study was exempt from full review by the internal review board.

\section{Patients and study design}

We used data for the years 1998 to 2008 from the National Health Insurance (NHI) Research Database, which contains data on all covered medical benefit claims for over 23 million people in Taiwan (approximately 97 percent of the island's population).

All patients with NPC (International Classification of Disease, Ninth Revision, Clinical Modification codes 147.0-147.9) who received curative treatment by radiotherapy or chemoradiotherapy between the years 1998 and 2000 were included. Patients with unclear treatment modality and incomplete physician data or treated by physicians with a very small caseload (less than 4 cases within 3 years) were excluded. Finally, 1225 patients treated by 98 radiation oncologist during this period were included.

Physicians were further sorted by their total patient volume using the unique physician identifiers in this database and by their caseload of NPC patients. The volume category cutoff points (high, medium, and low) were determined by sorting the 1225 patients into 3 groups of approximately equal size (4-16 cases [low], 17 -34 cases [medium], and $\geqq 35$ cases [high]) as previously described $[5,12,13]$.

These NPC patients were then linked to the death data extracted from the records covering the years 1998 to 2008.

\section{Measurements}

The key dependent variable of interest was the 10-year survival rate. The key independent variables were the NPC caseloads (low, medium, or high). Other physician characteristics included age ( $\leqq 40,41-50, \geqq 51$ years) and gender. Patient characteristics included age, gender, geographic location, treatment modality, severity of disease, and enrollee category (EC). The disease severity in each patient was assessed using the modified Charlson Comorbidity Index score, which has been widely used in recent years for risk adjustment in administrative claims data sets [14].

This study used EC as a proxy measure of socioeconomic status, which is an important prognostic factor for cancer patients $[15,16]$. Patients with NPC were classified into 4 subgroups: EC 1 (civil servants, full-time or regular paid personnel with a government affiliation), EC 2 (employees of privately owned institutions), EC 3 (self-employed individuals, other employees, and members of farmers' or fishermen's associations), and EC 4 (veterans, low-income families, and substitute service draftees) [17].

The hospitals were categorized by ownership (public, not-for-profit or for-profit), geographic location (Northern, Central, Southern, and Eastern Taiwan), and hospital type (medical center, regional hospital, and district hospital).

\section{Statistical analysis}

The SAS statistical package (version 9.2; SAS Institute, Inc., Cary, N.C.) and SPSS (version 15, SPSS Inc., Chicago, IL, USA) were used for data analysis. A two-sided value of $p<0.05$ was used to determine statistical significance.

The cumulative 10-year survival rates and the survival curves of each group were compared by the log-rank test. Survival was measured from the time of NPC diagnosis to the time of death. Cox proportional regression model and survival analysis with propensity score stratification were used to compare outcomes between different caseload size groups.

(1) Cox proportional hazards model The Cox proportional regression model was used to evaluate the effect of caseload on survival rate after adjusting for hospital type, surgeon characteristics, and patient demographics.

(2) Propensity score Propensity analysis was used to reduce the effect of selection bias on our hypothesis as described by Rosenbaum and Rubin [18-20]. Propensity score stratification replaces the many confounding factors that may be present in an observational study with a variable of these factors. To calculate the propensity score, patient characteristics in this study were entered into a logistic regression model predicting selection for high-volume surgeons. These characteristics included year in which the patient was diagnosed, age, gender, Charlson Comorbidity Index score, geographic area of residence, enrollee category, and treatment modality. The study population was then divided into five discrete strata on the basis of propensity score. The effect of caseload assignment on 10-year survival rate was analyzed within each quintile. The Mantel-Haenszel odds ratio was calculated in addition to the Cochran-MantelHaenszel $\chi^{2}$ statistic.

\section{Results}

A total of 423 patients (35\%) died out of 1225 patients who underwent curative treatment between 1998 and 2000. A total of 98 radiation oncologists were included. The characteristics of the physicians and patients are 
summarized in Tables 1 and 2 . The majority of the patients were male (72\%). Patients in the high-volume physician group were more likely to undergo radiotherapy, reside in Northern Taiwan, have lower comorbidity score, and better enrollee category than their counterparts in other groups. There were 74 radiation oncologists (76\%) in the low-volume group, 17 physicians (17\%) in the medium-volume group, and 7 (7\%) physicians in the high-volume group. The mean age of all physicians was $40 \pm 12$ years. There was no significant difference in age between these three caseload groups ( $p$ $=0.507$ ).

\section{Analysis using a Cox proportional hazards model}

The 10-year survival rate, by physician caseload group, is shown in Figure 1. The 10-year survival rates were $75 \%, 61 \%$, and $60 \%$ for low-, medium-, and high-volume surgeons, respectively $(p<0.001)$. Table 3 shows the adjusted hazard ratios calculated using the Cox proportional hazards regression model after adjusting for patient comorbidities, hospital type, and treatment modality. The positive association between survival and physician caseload remained statistically significant in multivariate analysis. Patients treated by high-volume
Table 2 Physician Characteristics $(n=98)$

\begin{tabular}{lcccc}
\hline \multirow{2}{*}{ Variable } & \multicolumn{3}{c}{ Physician caseload group } & \\
\cline { 2 - 4 } & $\begin{array}{c}\text { Low } \\
\mathbf{( 4 - 1 6 )}\end{array}$ & $\begin{array}{c}\text { Medium } \\
\mathbf{( 1 7 - 3 4 )}\end{array}$ & $\begin{array}{c}\text { High } \\
(\mathbf{3 5 - 1 5 2 )}\end{array}$ & $\boldsymbol{p}$ \\
\hline $\begin{array}{l}\text { Total no. physicians } \\
\text { Age(year) }\end{array}$ & 74 & 17 & 7 & \\
$\begin{array}{l}\text { Mean } \pm \text { SD } \\
\text { Gender }\end{array}$ & $39 \pm 13$ & $39 \pm 11$ & $45 \pm 13$ & \\
$\quad$ Male & $65(88)$ & $14(82)$ & $6(86)$ & \\
$\quad$ Female & $9(12)$ & $3(18)$ & $1(14)$ & \\
Caseload & & & & $<0.032$ \\
Mean \pm SD & $6 \pm 5$ & $24 \pm 6$ & $62 \pm 45$ & \\
\hline
\end{tabular}

Values are given as number (percentage).

Abbreviations: $\mathrm{SD}=$ standard deviation.

physicians had better survival rates (hazard ratio $[\mathrm{HR}]=$ 0.6; 95\% confidence interval $[\mathrm{CI}], 0.45-0.78 ; p<0.001$ ) after adjust other factors.

\section{Analysis using propensity scores}

Patients were stratified by propensity score and the effect of physician caseload on survival was assessed. The population was stratified into propensity quintiles

Table 1 Patient Characteristics in Different Caseload Groups $(n=1225)$

\begin{tabular}{|c|c|c|c|c|}
\hline \multirow[b]{2}{*}{ Variable } & \multicolumn{3}{|c|}{ NPC caseload group } & \multirow[b]{2}{*}{$p$} \\
\hline & $\begin{array}{c}\text { Low } \\
(4-16) \\
(n=424)\end{array}$ & $\begin{array}{c}\text { Medium } \\
(17-34) \\
(n=394)\end{array}$ & $\begin{array}{c}\text { High } \\
(35-152) \\
(n=407)\end{array}$ & \\
\hline Age & & & & 0.037 \\
\hline 35-44 years & 136(32) & $90(23)$ & $103(25)$ & \\
\hline $45-54$ years & $118(28)$ & $143(36)$ & $145(36)$ & \\
\hline $55-64$ years & 93(22) & $100(25)$ & $99(24)$ & \\
\hline $65-74$ years & $59(14)$ & $51(13)$ & $48(12)$ & \\
\hline$\geqq 75$ years & $18(4)$ & 10(3) & $12(3)$ & \\
\hline Gender & & & & 0.389 \\
\hline Male & $316(75)$ & $285(72)$ & $286(70)$ & \\
\hline Female & $108(25)$ & $109(28)$ & $121(30)$ & \\
\hline Charlson Comorbidity Index score & & & & $<0.001$ \\
\hline$<4$ & $216(51)$ & $229(58)$ & $274(67)$ & \\
\hline$\geqq 4$ & 208(49) & $165(42)$ & 133(33) & \\
\hline Treatment modality & & & & $<0.001$ \\
\hline Radiotherapy & $278(66)$ & $271(69)$ & $322(79)$ & \\
\hline Chemoradiotherapy & $146(34)$ & $123(31)$ & $85(21)$ & \\
\hline Geographic location & & & & $<0.001$ \\
\hline North & $266(63)$ & $240(61)$ & $317(78)$ & \\
\hline Central & $93(22)$ & $61(15)$ & $43(11)$ & \\
\hline Southern and Eastern & $65(15)$ & $93(24)$ & $47(11)$ & \\
\hline Enrollee category & & & & 0.008 \\
\hline EC 1-2 & $168(40)$ & $133(34)$ & $183(45)$ & \\
\hline EC 3 & $181(43)$ & $172(44)$ & $164(40)$ & \\
\hline EC 4 & 75(18) & $89(23)$ & $60(15)$ & \\
\hline
\end{tabular}

Values are given as number (percentage). 


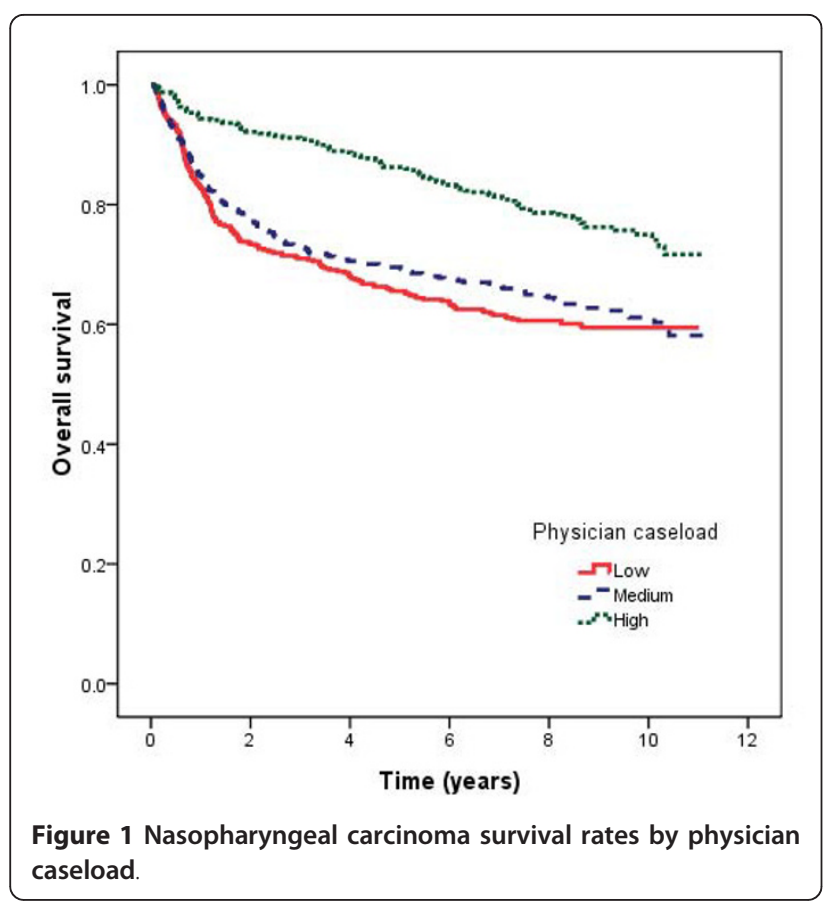

as previously described. Table 4 shows survival rates for both caseload groups after stratification. The percentage of patients treated by low/medium-volume physicians decreased from the first propensity quintile to the fifth as predicted by the propensity model. In each of the five strata, patients treated by high-volume physicians had a higher 10-year survival rate. The $p$ value for the Cochran-Mantel-Haenszel statistic for the difference in survival between patients treated by low/medium- and high-volume physicians, while controlling for propensity score, was $<0.001$, with fewer patients dying who were treated by high-volume physicians (adjusted odds ratio $=0.54,95 \% \mathrm{CI}, 0.41-0.7)$. The adjusted 10-year survival rates for low/medium- and high-volume physicians were $61 \%$ and $75 \%(p<0.001)$.

In summary, NPC patients treated by high-volume physicians had better survival. The robustness of this result was demonstrated by two different multivariate analyses, the Cox proportional regression model and stratification by propensity score.

\section{Discussion}

Using a Cox proportional hazards model and propensity score, the relative benefit of treatment by high-volume physicians over low/medium-volume physicians was evaluated in NPC. After controlling for patient characteristics and other variables in the Cox proportional regression model, the adjusted hazard ratio was 0.6 for
Table 3 Nasopharyngeal Carcinoma Survival Rate and Adjusted Hazard Ratios by Physician Caseload Groups and the Characteristics of the Patients and Providers ( $n=$ 1225)

\begin{tabular}{|c|c|c|c|}
\hline Variable & $\begin{array}{l}\text { Adjusted hazard } \\
\text { ratio }\end{array}$ & $95 \% \mathrm{Cl}$ & $p$ \\
\hline \multicolumn{4}{|l|}{ Physician characteristics } \\
\hline \multicolumn{4}{|l|}{ Physician volume } \\
\hline Low (3-17) & 1 & & \\
\hline Medium (17-53) & 0.884 & $0.70-1.16$ & 0.884 \\
\hline High (54-130) & 0.60 & $0.45-0.78$ & $\begin{array}{l}< \\
0.001\end{array}$ \\
\hline \multicolumn{4}{|l|}{ Physician age } \\
\hline$\leqq 40$ years & 1 & & \\
\hline 41-50 years & 1.22 & $0.97-1.52$ & 0.086 \\
\hline$\geq 51$ years & 0.78 & $0.59-1.02$ & 0.073 \\
\hline \multicolumn{4}{|l|}{ Hospital characteristics } \\
\hline \multicolumn{4}{|l|}{ Hospital ownership } \\
\hline Public & 1 & & \\
\hline Non-for-profit & 1.11 & $0.87-1.42$ & 0.414 \\
\hline For-profit & 0.94 & $0.65-1.36$ & 0.746 \\
\hline \multicolumn{4}{|l|}{ Hospital level } \\
\hline Medical center & 1 & & \\
\hline Regional hospital & 0.88 & $0.68-1.16$ & 0.368 \\
\hline District hospital & 1.25 & $0.77-2.03$ & 0.376 \\
\hline \multicolumn{4}{|l|}{ Patient characteristics } \\
\hline \multicolumn{4}{|l|}{ Patient gender } \\
\hline Female & 1 & & \\
\hline Male & 0.93 & $0.75-1.15$ & 0.509 \\
\hline \multicolumn{4}{|l|}{ Patient age } \\
\hline 35-44 years & 1 & & \\
\hline $45-54$ years & 1.15 & $0.89-1.49$ & 0.277 \\
\hline $55-64$ years & 1.10 & $0.83-1.45$ & 0.507 \\
\hline $65-74$ years & 1.12 & $0.81-1.56$ & 0.488 \\
\hline$\geqq 75$ years & 0.88 & $0.48-1.51$ & 0.675 \\
\hline \multicolumn{4}{|l|}{$\begin{array}{l}\text { Charlson Comorbidity } \\
\text { Index score }\end{array}$} \\
\hline$<4$ & 1 & & \\
\hline$\geqq 4$ & 1.28 & $1.04-1.56$ & 0.018 \\
\hline \multicolumn{4}{|l|}{ Treatment modality } \\
\hline Radiotherapy & 1 & & \\
\hline Chemoradiotherapy & 1.03 & $0.82-1.29$ & 0.784 \\
\hline \multicolumn{4}{|l|}{ Geographic location } \\
\hline North & 1 & & \\
\hline Central & 1.18 & $0.90-1.55$ & 0.242 \\
\hline $\begin{array}{l}\text { Southern and } \\
\text { Eastern }\end{array}$ & 1.30 & $1.00-1.70$ & 0.051 \\
\hline \multicolumn{4}{|l|}{ Enrollee category } \\
\hline EC 1-2 & 1 & & \\
\hline EC 3 & 1.35 & $0.71-2.55$ & 0.358 \\
\hline EC 4 & 1.04 & $0.86-1.26$ & 0.698 \\
\hline
\end{tabular}

$95 \% \mathrm{Cl}, 95 \%$ confidence interval. 
Table 4 10-year survival of NPC patients in different propensity score strata; low/medium-volume vs. high-volume physicians $^{\text {a }}$

\begin{tabular}{|c|c|c|c|c|c|c|c|}
\hline \multirow[t]{2}{*}{ Propensity score stratum } & \multicolumn{3}{|c|}{ Low/medium-volume physician group } & \multicolumn{3}{|c|}{ High-volume physician group } & \multirow[t]{2}{*}{$p$} \\
\hline & No. & $\%$ of stratum & Survival rate (\%) & No. & $\%$ of stratum & Survival rate (\%) & \\
\hline 1 & 193 & 79 & 56 & 51 & 21 & 75 & 0.004 \\
\hline 2 & 191 & 78 & 59 & 52 & 22 & 74 & 0.029 \\
\hline 3 & 173 & 70 & 57 & 74 & 30 & 75 & 0.013 \\
\hline 4 & 145 & 58 & 64 & 104 & 42 & 76 & 0.021 \\
\hline 5 & 116 & 48 & 69 & 126 & 52 & 76 & 0.28 \\
\hline Total & 818 & & 61 & 407 & 33 & 75 & $<0.001$ \\
\hline
\end{tabular}

a. Stratum 1 had the strongest propensity for low/medium physicians; stratum 5, for high-volume physicians.

b. Conchran-Mantel-Haenszel statistics; adjusted odds ratio $=0.54,95 \%$ confidence interval $=0.41-0.70$.

high-volume physicians, indicating that patients with NPC treated by high-volume physicians had a lower risk of death and were more likely to live longer. When analyzed by propensity score, the adjusted 10-year survival rate was $75 \%$ for patients treated by high-volume physicians and $61 \%$ for patients treated by low/mediumvolume physicians. Moreover, fewer patients treated by high-volume physicians died. The results of both forms of analyses led to the conclusion that the 10-year survival rates for patients with NPC treated by high-volume physicians were significantly better.

Previous studies have evaluated the benefits of high hospital and physician volume on the outcomes of cancer treatment. In head and neck cancer, Lin et al. reported that physician volume (not hospital volume) was associated with oral cancer survival rates [5]. In our series, we also found a better 10-year survival rate associated with treatment by high-volume physicians.

The quality of the risk-adjustment technique in analyzing administrative information is an important issue. In the first part of this study, a Cox proportional hazard model was used to compare the effects of high volume versus low/medium volume on survival rate. We found treatment by high-volume physicians was significantly associated with lower adjusted hazard ratio for death. Patients treated by high-volume physicians were found to have a $40 \%$ lower risk of death after adjusting for comorbidities and other confounding factors. However, there was some difference in age and clinical condition between caseload groups. In the second part of our series, propensity score was used to stratify patients into five strata with similar propensity score in order to reduce the effect of selection bias on caseload groups [19-21]. Patients treated by high-volume physicians were found to have a $14 \%$ relative improvement in adjusted 10 -year survival rate $(p<0.001)$.

Although NPC patients may be followed up in a team consisting of otolaryngologist, radiation oncologists, hematology oncologists, and radiologists, the cornerstone of treatment of NPC relied on the successful eradication of disease by radiotherapy. In order to explore the caseload effect of radiotherapy on NPC survival, we calculated the caseload volume of radiation oncologists. In agreement with previous volume-outcome studies, our results indicated that increased caseload of radiation oncologists is associated with improved outcomes after other factors.

Several hypotheses relating to the volume-outcome relationship have been proposed. The "practice makes perfect" concept suggests that increased caseload may help physicians or hospital staff improve the execution of treatment procedures, such as planning the radiation field and manipulation of the radioactive source of teletherapy units. The role of surgery in the treatment of NPC is limited, and carefully defining the planning target volume with the aid of CT or MRI images is important for radiotherapy or concurrent chemoradiotherapy in NPC. A high-volume team may be more adept at administering a radiation dose, with or without a booster dose, that balances the benefit of successful locoregional control against the risk of radiation toxicity.

Previous study reported that high-volume physicians use effective treatment and strategies more often than do low-volume physicians [22]. In breast cancer series, high-volume surgeons adopted a multi-disciplinary approach whereas low-volume surgeons were less likely to interact with oncologists or attend multi-disciplinary meetings [23]. Use of multidisciplinary approaches may account for the better outcomes achieved by highvolume physicians. Possibly, low-volume physicians do not always follow the international guidelines for NPC treatment.

The "selective referral hypothesis" postulates that healthier patients or patients with early-stage disease tend to be referred to high-volume physicians. The referral system in Taiwan is weakly enforced, and people are free to choose any physician. Because official performance information to help consumers select healthcare providers is not available, patients choose physicians with better reputations or more successful physicians after 
consulting with their relatives and friends [4]. Selective referral bias may also result from the referral of more curable patients to high-volume physicians. Patients not seeking curative treatment or for whom curative treatment is not possible may continue to receive their care from low-volume physicians.

Our study revealed some issues that may be useful for policy makers. Research is needed to identify the differences in care and treatment strategy between low-, medium-, and high-volume physicians. In our study, nearly $33 \%$ of patients were treated by 7 high-volume radiation oncologists. The viewpoints of high-volume physicians may influence the development of effective protocols and practice guidelines for the majority of clinical situations. The treatment strategies of high-volume physicians should be analyzed and adopted throughout the country to improve survival rates.

Our study has several limitations. First, we could not assess the relationship of caseload to NPC stage because this information was not available from the database. However, Begg et al., using a SEER-Medicare linked database, reported that cancer stage and patient age were independent of caseload volume [24]. Instead of cancer-specific survival rates, overall survival rate was used, because it was not possible to determine causespecific mortality based on the registry data. Previous study by Roohan et al. showed no significant difference between survival models for all-cause mortality and breast cancer mortality [25]. Given the robustness of the evidence and statistical analysis in this study, these limitations are unlikely to compromise our results.

In summary, our findings support the conclusion that provider volume affects survival outcome in NPC. Analysis using a Cox proportional hazard model and propensity score found an association between high-volume physicians and improved 10-year survival rate in patients with NPC. Analysis of the treatment strategies adopted by high-volume physicians may improve overall survival rate.

\section{Conflict of interest}

The authors declare that they have no competing interests.

\section{Acknowledgements \\ This study is based in part on data from the National Health Insurance Research Database provided by the Bureau of National Health Insurance Department of Health and managed by the National Health Research Institutes (Registry number 99018). The interpretation and conclusions contained herein do not represent those of the Bureau of National Health Insurance, Department of Health, or National Health Research Institutes.}

\section{Author details}

'Department of Otolaryngology, Buddhist Dalin Tzu Chi General Hospital, Chiayi, Taiwan. ${ }^{2}$ Department of Oral and Maxillofacial Surgery, Buddhist Dalin Tzu Chi General Hospital, Chiayi, Taiwan. ${ }^{3}$ Department of Radiation
Oncology, Buddhist Dalin Tzu Chi General Hospital, Chiayi, Taiwan. ${ }^{4}$ Department of Hematology Oncology, Buddhist Dalin Tzu Chi General Hospital, Chiayi, Taiwan. ${ }^{5}$ Division of Plastic Surgery, Department of Surgery, Buddhist Dalin Tzu Chi General Hospital, Chiayi, Taiwan. ${ }^{6}$ School of Medicine, Tzu Chi University, Hualien, Taiwan. ${ }^{7}$ Community Medicine Research Center and Institute of Public Health, National Yang-Ming University, Taipei, Taiwan.

\section{Authors' contributions}

LCC, CSH and HSK developed the ideas for these studies, performed much of the work, and drafted the manuscript. CSH, CP, LCC, HTT and HSK revised the manuscript. LMS, SYC, CP, CWY and LHY designed the study, managed and interpreted the data. LCC performed the statistical analysis. All authors read and approved the final manuscript.

Received: 27 February 2011 Accepted: 11 August 2011

Published: 11 August 2011

\section{References}

1. Luft HS, Bunker JP, Enthoven AC: Should operations be regionalized? The empirical relation between surgical volume and mortality. $N$ Engl J Med 1979, 301:1364-1369.

2. Halm EA, Lee C, Chassin MR: Is volume related to outcome in health care? A systematic review and methodologic critique of the literature. Ann Intern Med 2002, 137:511-520.

3. Luft Harold S, Hunt Sandra S, Maerki SC: The volume-outcome relationship: practice-makes-perfect or selective-referral patterns? Health Serv Res 1987, 22:157-582.

4. Cheng $\mathrm{SH}$, Song HY: Physician performance information and consumer choice: a survey of subjects with the freedom to choose between doctors. Qual Saf Health Care 2004, 13:98-101.

5. Lin CC, Lin HC: Effects of surgeon and hospital volume on 5-year survival rates following oral cancer resections: the experience of an Asian country. Surgery 2008, 143:343-351.

6. Lin CS, Lee HC, Lin CT, Lin HC: The association between surgeon case volume and hospitalization costs in free flap oral cancer reconstruction operations. Plast Reconstr Surg 2008, 122:133-139.

7. Department of Health: The Executive Yuan: Cancer registry annual report. Republic of China 2004

8. Al-Sarraf M, LeBlanc M, Giri PG, Fu KK, Cooper J, Vuong T, Forastiere AA, Adams G, Sakr WA, Schuller DE, Ensley JF: Chemoradiotherapy versus radiotherapy in patients with advanced nasopharyngeal cancer: phase III randomized Intergroup study 0099. J Clin Oncol 1998, 16:1310-1317.

9. Begg CB, Riedel ER, Bach PB, Kattan MW, Schrag D, Warren JL, Scardino PT: Variations in morbidity after radical prostatectomy. N Engl J Med 2002, 346:1138-1144.

10. Begg CB, Cramer LD, Hoskins WJ, Brennan MF: Impact of hospital volume on operative mortality for major cancer surgery. JAMA 1998, 280:1747-1751.

11. Sosa JA, Bowman HM, Gordon TA, Bass EB, Yeo CJ, Lillemoe KD, Pitt HA Tielsch JM, Cameron JL: Importance of hospital volume in the overall management of pancreatic cancer. Ann Surg 1998, 228:429-438.

12. Laks MP, Cohen T, Hack R: Volume of procedures at transplantation centers and mortality after liver transplantation. N Engl J Med 2000, 342:1527.

13. Goodney PP, Stukel TA, Lucas FL, Finlayson EV, Birkmeyer JD: Hospital volume, length of stay, and readmission rates in high-risk surgery. Ann Surg 2003, 238:161-167.

14. Deyo RA, Cherkin DC, Ciol MA: Adapting a clinical comorbidity index for use with ICD-9-CM administrative databases. J Clin Epidemiol 1992, 45:613-619.

15. Braaten $T$, Weiderpass $E$, Lund E: Socioeconomic differences in cancer survival: the Norwegian Women and Cancer Study. BMC Public Health 2009, 9:178.

16. Kwok J, Langevin SM, Argiris A, Grandis JR, Gooding WE, Taioli E: The impact of health insurance status on the survival of patients with head and neck cancer. Cancer 2010, 116:476-485.

17. Chen CY, Liu CY, Su WC, Huang SL, Lin KM: Factors associated with the diagnosis of neurodevelopmental disorders: a population-based longitudinal study. Pediatrics 2007, 119:435-443.

18. Joffe MM, Rosenbaum PR: Invited commentary: propensity scores. Am J Epidemiol 1999, 150:327-333. 
19. Rubin DB: Tasks in statistical inference for studying variation in medicine. Med Care 1993, 31:YS103-110.

20. Rubin DB: Estimating causal effects from large data sets using propensity scores. Ann Intern Med 1997, 127:757-763.

21. D'Agostino RB Jr: Propensity score methods for bias reduction in the comparison of a treatment to a non-randomized control group. Stat Med 1998, 17:2265-2281.

22. Thiemann DR, Coresh J, Oetgen WJ, Powe NR: The association between hospital volume and survival after acute myocardial infarction in elderly patients. N Engl J Med 1999, 340:1640-1648.

23. Stefoski Mikeljevic J, Haward RA, Johnston C, Sainsbury R, Forman D: Surgeon workload and survival from breast cancer. Br J Cancer 2003, 89:487-491.

24. Begg CB, Cramer LD, Hoskins WJ, Brennan MF: Impact of hospital volume on operative mortality for major cancer surgery. JAMA 1998, 280:1747-1751.

25. Roohan PJ, Bickell NA, Baptiste MS, Therriault GD, Ferrara EP, Siu AL: Hospital volume differences and five-year survival from breast cancer. Am J Public Health 1998, 88:454-457.

doi:10.1186/1748-717X-6-92

Cite this article as: Lee et al: Survival rate in nasopharyngeal carcinoma improved by high caseload volume: a nationwide population-based study in Taiwan. Radiation Oncology 2011 6:92.

\section{Submit your next manuscript to BioMed Central} and take full advantage of:

- Convenient online submission

- Thorough peer review

- No space constraints or color figure charges

- Immediate publication on acceptance

- Inclusion in PubMed, CAS, Scopus and Google Scholar

- Research which is freely available for redistribution

Submit your manuscript at www.biomedcentral.com/submit
C Biomed Central 